\title{
Die Schafe werden sich in Wölfe verwandeln.
}

\author{
Von Oscar Holtzmann in GieBen.
}

In dem abschließenden eschatologischen Kapitel der Didache heißt

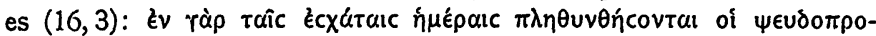

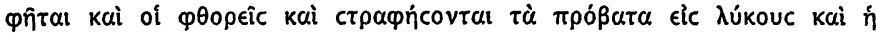

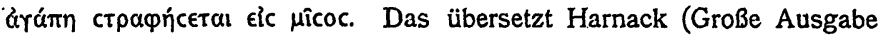
von 1884, S. 61): „Denn in den letzten Tagen werden sich die Pseudopropheten und Verderber vermehren und werden die Schafe in Wölfe verkehren, und die. Liebe wird sich in $\mathrm{Ha}$ berkehren." Dagegen lautet die Übersetzung von Drews (Hennecke, NT. Apokr S. 193): "Denn in den letzten Tagen werden der falschen Propheten und Verderber viele werden, und die Schafe werden sich in Wölfe und die Liebe wird sich in $\mathrm{Haß}$ verkehren."

Harnack sieht also in den Pseudopropheten und Verderbern das tätige Subjekt, in den Schafen das leidende Objekt bei der Verwandlung der Schafe in Wölfe. Drews versteht das Verbum cтрa甲ńcєc $\theta \alpha l$ beidemal reflexiv (sich verkehren) und betrachtet $\tau \grave{\alpha}$ ipó $\beta \alpha \tau \alpha$ als Sub-

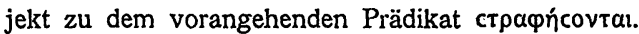

Für beide Übersetzungen lassen sich Gründe geltend machen. Für Hànacks Übersetzung spricht die pluralische Form, die in der Regel nicht steht, wenn der Plural eines Neutrums das Subjekt ist. So heißt

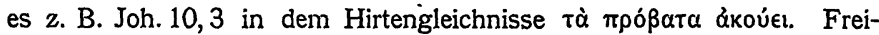
lich erleidet diese Regel sehr häufig Ausnahmen. Das zeigt gleich die Fortsetzung des johanneischen Hirtengleichnisses. V. 4. 5 heißt es:

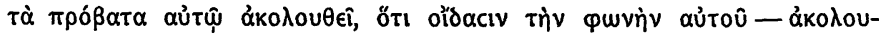

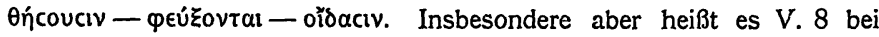

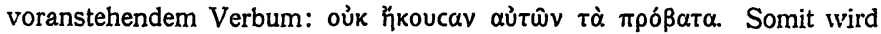

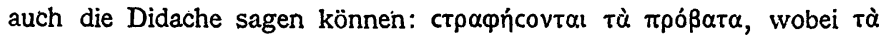
$\pi \rho o ́ \beta \alpha \tau \alpha$ als Subjekt zu denken ist.

Weiter könnte die Wortstellung für Harnacks Übersetzung sprechen. 
In dem letzten Glied steht das Subjekt í ărámn voraus. Wenn das Verbum im vorangehenden Glied ebenso wie in diesem letzten übersetzt werden soll, so könnte man, schon um Zweifel auszuschließen, auch dieselbe Wortstellung erwarten. Aber auch in ersten Teil des dreigliedrigen Satzes ist der vorangehenden Zeitbestimmung zuliebe die

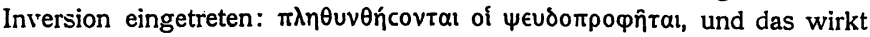
im zweiten Glied noch nach. Die Rückkehr zu der gewöhnlichen Wortstellung im dritten Glied dürfte ihren Anlaß in der nachträglichen

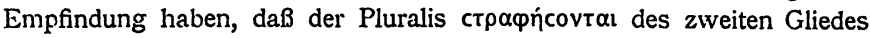
zu dem Subjekt $\mathfrak{\eta}$ àrárn als einem Singularis nićhit recht paßt. Oder es tritt nach einem öfters beobachteten Gesetz der Rhetorik am Schluß des Satzes die ruhige regelmäßige Wortstellung ein nach der vorausgehenden, pathetisch wirkenden Inversion; das ganze Kapitel 16 ist ja eine Mahnrede. Aus dieser Rückkehr vom Pathos zur Ruhe ergibt sich

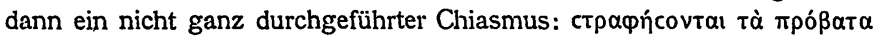

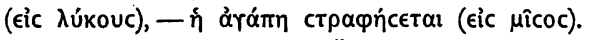

Entscheidend für die Übersetzung von Drews ist aber gerade die rasche Folge der beiden Formen desselben Verbums in demselben

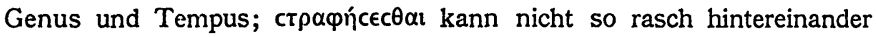
zwei verschiedene Bedeutungen haben; der passive oder reflexive Sinn, der im letzten Glied über alle Zweifel erhaben ist, muß auch im vorangehenden Gliede zu Recht bestehen. Es kommt hinzu, daß für die aktive Bedeutung (verwandeln) die aktive oder mediale Form des Verbums zur Verfügung standen. Der passiven Form entspricht allein die passive Bedeutung. Der Satz heißt also: „Die Schafe werden sich in Wölfe verwandeln."

Aber den Sinn des Satzes in der Didache hat Harnack durch seine Übersetzung richtig wiedergegeben, wenn auch diese Übersetzung mit der grammatischen Form des Satzes nicht übereinstimmt. In der Anmerkung sagt er: „Dies Bild nur hier; zum Gedanken s. Mt 24, 10:

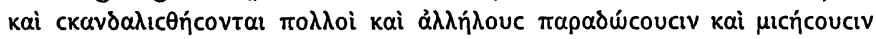
ả $\lambda \lambda \dot{n} \lambda$ ouc (Mt 7, 15 hat wohl auf die Fassung in der Didache eingewirkt)." Es ist zweifellos, daß die Umwandlung der Schafe in Wölfe an unserer Stelle als Bild gedacht ist für die geistige Umwandlung friedlicher in bösartige Menschen. Das ist ja im dritten Satzglied ohne Bild ausgesprochen. Und aus dem ersten Satzglied ist zu erschließen, daß diese geistige Umwandlung als Wirkung des Auftretens der Pseudopropheten und Verderber gedacht werden soll.

Aber das Bild von der. Verwandlung der Schafe in Wölfe ist doch sehr auffallend. Die von Harnack angeführte Stelle der Bergpredigt 
Mt. 7, 15 warnt freilich vor falschen Propheten und redet auch von Wölfen und Schafen. Aber da sind es die falschen Propheten, die ihre Wolfsnatur im Schafspelz verstecken, um sich in die Herde einzuschleichen und die Schafe leichter rauben zu können, wie das eine äsopische Fabel erzähit, über deren Ursprungszcit ich nicht urteilen will. Älter ist wohl die auch bei Babrius erhaltene Form, wonach der Wolf eine Zeitlang den Hund bei der Herde spielt. Jedenfalls verwandelt sich hier nicht einmal der Wolf in ein Schaf; gerade die Wolfsnatur bleibt ja unter dem Schafsfell crhalten. Hier aber wird erwartet, daß sich Schafe in Wölfe verwandeln. Also es stimmt nicht recht, wenn Drews zu der Stelle (Handbuch z. NT. Apokr S. 283) bemerkt: „vgl. MIt 7,15, wo das Bild in umgekehrter Weise verwendet ist."

Er fügt hinzu: „vgl. auch Mt 10,16.“ Da sagte Jesus zu seinen Jüngern: ich sende euch wie Schafe in die Mitte der Wölfe. Auch das ist ein Bild, das sich ebenso anderswo findet. Nach Herodot IV, 149 sagte ein gewisser Theras zu seinem Sohne, der in der Heimat zurückblieb, die der Vater verlassen wollte, „er werde inn wie ein Lamm unter den Wölfen zurücklassen“. Hërodot fügt hinzu: „nach diesem Wort erhielt der junge Mensch den Namen Oiolykos". Auch dieses Wort spricht in keiner Weise von einer Verwandlung der Schafe in Wölfe. Die Gefahr, die den Schafen durch Wölfe droht, wird außer Mt 10, 16 noch mehrfach im NT. erwähnt: im johanneischen Hirtengleichnis (Joh 10,12) und in der Rede des Paulus zu Milet (Act 20, 29). Mit dem Bilde der Didache hat das alles nichts zu schaffen.

"Die Schafe werden in Wölfe verwandelt werden." Das heißt verallgemeinert: $z$ ahme Tiere werden $z \mathrm{u}$ wilden Tieren. Nun finden wir in der Bibel und außerhalb der Bibel eine zweifache Anschauung yon Verwandlung der Tiere. In der Urzeit, sagt die eine Anschauung, sind die jetzt wilden Tiere zahm gewvesen, und in der Endzeit, sagt die andere Anschauung, werden sie wieder zahm werden. Die erste Anschauung liegt sicher vor Gen 3, 14. 15. Erst der Fluch Gottes nach dem Sündenfall bringt.Feindschaft zwischen die Schlange und den Menschen und läßt die Schlange dem Menschen gefährlich werden. Auf alle Tiere erweitert findet sich dann der Gedanke bei Philo (quaest. et solut. in Genesin I, 18 zu Gen 2, 19): ego putarem, quod nunc homini ob malum sibi insitum inimica et oppugnantia sint animalia tam terrestria quam volatilia; primo vero (homini) omnino virtutibus exornato tamquam socios belli in subsidium fuisse et amicos familiares ex natura cicuritate praeditos. Aber das ist keinestvegs ein Fündlein und 
eine individuelle Anschauung Philos. Bereschith rabba c. 25 heißt es: "nachdem der Mensch gesündigt hatte, lehnten sie sich wider ihn auf: die Kuh wollte dem Pflüger nicht mehr Folge leisten." Dillmann hat in seinem Genesiskommentar (6. Aufl., S. 47) diese Vorstellung von den Frieden der Urzeit bei vielen Völkern nachgewiesen. Das spieit fort bis in die Literatur der Gegenwart. In zweiten Aufzug seines Herzog Ernst läßt Uhland den Bischof Warmann das selige Paradies schildern: „Der Wolf hat sich zum Lamm hingestreckt, der Geier nistet mit der frommen Taube." Als dieser Zustand aufhörte, da haben sich zahme Tiere in wilde verwandelt.

Was in der Urzeit verloren ging, das soll die Endzeit wiederbringen. Nach Hosea 2, 20 schließt Jahve jenes Tags für sein Volk einen Vertrag mit den wilden Tieren und den Vögeln unter dem Himmel und dem Gerrürm auf der Erde. Aber am eindrucksvollsten ist die Schilderung der Endzeit bei Jesaja (11,6-9): „Der Wolf wird neben dem Lamme wohnen und der Parder neben dem Böcklein lagern, und Rind und Löwe und Mastvieh werden zusammen weiden, und ein kleiner Knabe sie leiten; Kuh und Bärin werden weiden und ihre Jungen nebeneinander lagern, und der Löwe wird sich, wie die Rinder, von Stroh nähren. Der Säugling wird an der Höhle der Otter spielen und der Entwöhnte seine Hand auf das Auge der Natter legen. Sie werden keinen Schaden und kein Verderben zufügen in meinem ganzen heiligen Bergland" (Kautzsche Übersetzung). Sehr ausführlich schildert Philo, wie Gott in der Endzeit die wilden Tiere zähmen und dem Menschen gefügig machen wird (de praem. et poen. 15); die Sibylle (III, 787-794) hat die Weissagung des Jesaja fast Wort für Wort in griechische Verse gebracht; im syrischen Baruch 73, 6 heißt es: „Und die wilden Tiere werden aus dem Walde kommen und den Menschen zu Diensten sein; und die Nattern und Drachen werden aus ihren Löchern hervorkriechen, um sich den kleinen Kindern zur Verfügung zu stellen. “ Daß die Wildheit der Tiere etwas Krankhaftes, vom Schöpfer nicht ursprünglich Gervolltes ist, deutet Bereschith rabba 95 an, wo es in der Schilderung der Endzeit heißt: "auch die Tiere werden geheilt von der Mordgier, die jetzt in ihnen herrscht, und dem Blutdurst, so daß das Lamm sich nicht mehr vor dem Wolfe zu fürchten hat und alle Tiere nur Pflanzen fressen." Auf heidnischem Boden findet sich eine ähnliche Anschauung in Vergils vierter Ekloge, wo es von der "seligen Zeit der Zukunft heißt $(\mathrm{V}, 22)$ : nec magnos metuent armenta leones. Das ist allerdings wohl so $\mathrm{zu}$ verstehen, daß die großen Löwen dann ausgestorben sein werden. V. 24 fügt hinzu: "occidet et serpens". Aber auch so wird 
cin Friedenszustand in der Tierwelt und zwischen Menschen und Tier erhofft.

Usener (Religionsgeschichtliche Untersuchungen III, S. $200 \mathrm{ff}$.) hat griechische Sagen zusammengestellt, wonach an heiligen, gottgeweihten Orten wilde Tiere entweder nicht vorkommen oder beim Betreten des hciligen Bodens ihre Wildheit verlieren: so in Kreta, im Eschenhain von Klaros bei Kolophon, am Timavus in Istrien. Nun ist auch das Paradies als der Garten Gottes vorgestellt, in dem sich Jahve in der Kühle des Abends ergeht (Ez 28,13; Gen 3,8); auch in diesem Gottesgarten sind die wilden Tiere friedlich und zahm. Das Paradies steht aber am Ende wie am Anfang der Weltgeschichte; die letzte Seligkeit wird sehr oft als Wiederbringung des Paradieses gedacht. Das ist nachweisbar seit Jes 51, 3 (s. meine Zeitgeschichte ${ }^{2}$ S. 406 f.; Greßmann, Urspr. der israel.-jüd. Eschatologie S. 198 f.). Es entspricht also einer weitverzweigten Vorstellung, wenn in der Gotteswelt der Urzeit und in der Gotteswelt der Endzeit die wilden Tiere zahm sind. Auch im Neuen Testament hat sich noch eine fast nur angedeutete Beziehung auf diese Vorstellung erhalten. Der. mit Gottes Geist erfüllte, vom Satan versuchte Messias lebt in der Wüste mit den Tieren zusammen, und die

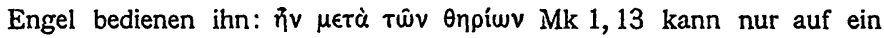
friedliches Zusammenleben hinweisen. Der Messias genießt schon im voraus den Frieden des Gottesreiches.

Zahme Tiere, die in der Urzeit des Menschen Freunde waren, sind nach der Vertreibung aus dem Paradiese des Menschen Feinde geworden. Es ist das nur ein Zug in dem Gesamtbilde des Elendes, das seit dem Sündenfall über die Welt hereinbricht. Nach der Schilderung der Genesis treffen das Weib seitdem die Schmerzen der Geburt, der Mensch leidet unter der Mühsal seiner Arbeit und verfällt schließlich dem Tod. Aber auch der Acker ist seitdem verflucht und trägt Dorn und Distel (Gen 3, 16-19). Das wird Bereschith rabba 25 dahin erklärt, daß auch die Furche sich jetzt gegen den Pflüger auflehnt und sich nicht mehr von ihm ziehen lassen will. Ja nach c. 5 dieses Kommentars brachte die Erde erst jetzt von sich aus schädliche Insekten hervor.

Nun haben diese ersten Sündenstrafen den Menschen nicht gebessert. Im Gegenteil. Dem Tode verfallen ist der Mensch schwächer zum Guten; das Judentum bildet die Theorie der Erbsünde aus; die Sünde haftet gewissermaßen an dem vergänglichen Leibe des Menschen (IV Esra 3, 7.8.21. 22; 4, 30; 7, 118; 14, 14; Baruch syr. 17, 2. 3; 48, 42 bis $47 ; 49,3 ; 54,15.19 ; 56,5-15$; Rom $5,12.14$; 1 Kor 15,22$)$. Damit hängt nun die Theorie zusammen von der periodisch fortschreiten- 
den Verschlechterung der Welt. Sie tritt uns zuerst auf heidnischem Boden entgegen und ist dann in demselben Bild auch vom Judentum übernommen worden. Bei ihrer engen Verwandtschaft mit der Lehre von der Erbsünde muß die Möglichkeit offen gelassen werden, daß auch diese Lehre vom Judentum aus fremder Quelle übernommen worden ist.

Bei Hesiod (Werke und Tage $109 \mathrm{ff}$.), Aratus (Phainomena 105ff.) und Ovid (Metam. I, 89 ff.) finden wir die Erzählung von den Weltaltern, die ihr Symbol in verschiedenen Metallen haben. Das Folgende ist immer schlechter als das Vorangehende, zuerst das Gold, dann das Silber, dann das Erz, dann das Eisen. So ist es bei Ovid, der uns hier wohl das Ursprüngliche erhalten hat, wenn er gleich die jüngste Quelle ist. Bei Hesiod ist die Reihe durchbrochen, sofern zwischen Erz und Eisen das Heroenzeitalter eingeschoben ist, das gerechter und besser ist als das eherne (158); Aratus lag vielleicht dieser Einschub in die ursprüngliche Darstellung schon vor, und er hat deshalb mit dem ehernen Alter abgeschlossen. Ob die Griechen die letzte Quelle für diese Mythologie sind, ist fraglich. In dem allerdings späten, aber auf alte Quellen zurückgehenden Buch Bahman-Yast wird von einem Perser der Baum geschildert, dessen vier Zweige aus Gold, Silber, Erz und Eisen bestehen (c. 1). Dieselbe Anschauung findet sich nun aber auch im Buch Daniel (2, 32-35.37-45). Der Koloß, von dem Nebukadnezar träumt, bedeutet die Weltreiche, die einander ablösen, immer das folgende schlechter und furchtbarer als das vorangehende, und auch hier sind die Symbole Gold, Silber, Erz, Eisen (und Ton). Die Weltentwicklung geht abwärts vom Hohen und Edeln zum Niedrigen und Gemeinen. In das Buch Daniel paßt freilich dieses Bild nur sehr schlecht: denn es stellt ja schon Nebukadnezar als den Ausbund alles Bösen dar. Aber dem Verfasser war es trotzdem unzweifelhaft, daß die Welt immer schlimmer geworden sei bis auf das eiserne und tönerne Zeitalter, in dem er lebt.

Und nun hat er dafür noch ein anderes Bild bereit. Er schildert die vier Weltreiche auch als vier Tiere, von denen immer das folgende schrecklicher ist als das vorangehende (7,2-7). Das erste, ein Löwe mit Adlerflügeln, erhält Menschenverstand; das zwveite, ein Bär, wird schon beauftragt, viel Fleisch $z u$ fressen; das dritte ist dann ein vierköpfiger und mit vier Flügeln ausgestatteter Panther; aber das vierte Tier ist ,fürchterlich, schrecklich und außerordentlich stark, mit gewaltigen eisernen Zähnen', mit Füßen;" die alles zertreten, was es nicht frißt und zermalmt, und mit einem Schmuck von zehn Hörnern. Diese Tiere sind dem Buch Daniel das Bild für den immer schlimmer werdenden Druck der Weltmächte. Aber erfunden hat der Verfasser das Bild so 
wenig wie das anderc von den vier Metallen. Und doch ist ein wesentlicher Unterschied zwischen beiden Bildern. Die vier Metalle drücken nur die Abstufung im Werte der aufeinander folgenden Zeiten und Reiche aus. Die vier Tiere stellen das allmähliche Schlimmerwerden der Welt an einer Art Entwicklungsreihe aus dieser Welt selber dar. Es ist nicht genug, daß der Friede in und mit der Tierwelt aufgehört hat, als die Menschen das Paradies verlassen mußten: der fortdauernden Verschlechterung der Menschen entspricht die fortdauernde Verwilderung der Tiere. Aus einer solchen Anschauung heraus, die freilich der Wirklichkeit der Dinge im ganzen keck widerspricht, ist das Bild von den vier Tieren des Daniel erwachsen. Richtig an dieser Anschauung ist, daß ganze Gattungen großer pflanzenfressender Tiere den Zähnen der Fleischfresser allmählich erlagen. Das Recht des Stärkeren müßte $\mathrm{zu}$ einer immer sich steigernden Verwilderung der Tierwelt führen, wenn hier bloß die physischen Kräfte in Betracht kämen.

$\mathrm{Zu}$ der ganzen hier vorgeführten Geschichtsbetrachtung gehört die Erwartung einer höchsten Steigerung des Bösen am „Ende der Tage“. Davon spricht die Didache. ausführlich, in dem Zusammenhang, in dem das Wort von der Umwandlung der Schafe in. Wölfe begegnet $(16,3.4)$. Von dieser letzten schweren Zeit redet die jüdisch-christliche Apokalyptik immer wieder. Es genügt an die Parusierede bei den Synoptikern und an die Plagen in der johanneischen Apokalypse zu erinnern. Aber diese Erwartung ist viel älter. Und schon in ihrem ältesten Bestand redet sie gern von einer Not durch gefährliche Tiere. $\mathrm{Da}$ ist von Löwe, Bär und Schlange die Rede (Amos 5,19), von Wolf, Panther und Basilisk (Jer 5,$6 ; 8,17)$ von Heuschrecken (Joel 1.2), von Fliege und Biene (Jes 7,18.19). Selbst ein so aufgeklärter Philosoph wie Philo läßt Schwärme von Wespen im letzten Entscheidungskampf die Hauptarbeit tun (de præm. et pœn. 16). Dem Apokalyptiker Johannes verkörpert sich nicht bloß aller Schrecken der letzten Zeit in einem gewaltigen Tier (13); er weiß von Heuschrecken mit Frauenhaaren, Löwenzähnen und Eisenpanzern, mit Schwänzen gleich Skorpionen und Stacheln (9, 8-10); er weiß von Pferden mit feuerspeienden Löwenköpfen und Schweifen aus Schlangen $(9,17.19)$. Diese Tierplagen gehören notwendig zum Bilde der letzten Not.

In diese eschatologische Bilderwelt hinein gehört nun meines $\mathrm{Er}$ -

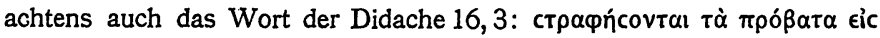
$\lambda$ úkouc. Es ist eine der erwarteten Plagen, die nach der volkstümlichen Erwartung dem Zusammenbruch dieser Welt vorhergehen. Der Verfasser wollte sie allerdings wahrscheinlich ins Geistige umgedeutet 
wissen: aus friedlichen werden gefährliche, bösartige Menschen werden. Diese Deutung läßt er erraten, indem er das Satzglied von falschen Propheten und Verderbern vorausschickt und das andere Satzglied von der Umwandlung der Liebe in $\mathrm{HaB}$ anschließt. Aber um ein frei erfundenes Bild oder um eine dem Augenblick entsprechende freie Umgestaltung eines Herrnwortes handelt es sich meines Erachtens hier nicht; vielmehr haben wir in dem Wort von der Umivandlung der Schafe in Wölfe den Ausdruck eines uralten Glaubens. Wir können nicht entscheiden, wie weit ihn der Verfasser noch. geteilt hat. 